\title{
Dark Solitons in Bose-Einstein Condensates
}

\author{
S. Burger, K. Bongs, S. Dettmer, W. Ertmer, and K. Sengstock \\ Institut für Quantenoptik, Universität Hannover, 30167 Hannover, Germany \\ A. Sanpera, ${ }^{1}$ G. V. Shlyapnikov, ${ }^{1,2,3}$ and M. Lewenstein ${ }^{1}$ \\ ${ }^{1}$ Institut für Theoretische Physik, Universität Hannover, 30167 Hannover, Germany \\ ${ }^{2}$ FOM Institute for Atomic and Molecular Physics, Kruislaan 407, 1098 SJ Amsterdam, The Netherlands \\ ${ }^{3}$ Russian Research Center Kurchatov Institute, Kurchatov Square, 123182 Moscow, Russia
}

(Received 27 October 1999)

Dark solitons in cigar-shaped Bose-Einstein condensates of ${ }^{87} \mathrm{Rb}$ are created by a phase imprinting method. Coherent and dissipative dynamics of the solitons has been observed.

PACS numbers: 03.75.Fi, 32.80.Pj

The realization of Bose-Einstein condensation (BEC) of weakly interacting atomic gases [1] strongly stimulates the exploration of nonlinear properties of matter waves. This supports the new field of nonlinear atom optics, e.g., four wave mixing in BEC's [2], as well as the study of various types of excitations. Of particular interest are macroscopically excited Bose condensed states, such as vortices and solitons. Vortices, well known from the studies of liquid helium [3], have recently been observed in two component gaseous condensates [4].

Solitonlike solutions of the Gross-Pitaevskii equation are closely related to similar solutions in nonlinear optics describing the propagation of light pulses in optical fibers. Here, bright soliton solutions correspond to short pulses where the dispersion of the pulse is compensated by the self-phase modulation, i.e., the shape of the pulse does not change. Similarly, optical dark solitons correspond to intensity minima within a broad light pulse [5].

In the case of nonlinear matter waves, bright solitons are expected only for an attractive interparticle interaction ( $s$-wave scattering length $a<0$ ) [6], whereas dark solitons, also called "kink-states," are expected to exist for repulsive interactions $(a>0)$. Recent theoretical studies discuss the dynamics and stability of dark solitons [7-10], as well as concepts for their creation [11-13].

Conceptually, solitons as particlelike objects provide a link of BEC physics to fluid mechanics, nonlinear optics, and fundamental particle physics.

In this Letter we report on the experimental investigation of dark solitons in cigar-shaped Bose-Einstein condensates in a dilute vapor of ${ }^{87} \mathrm{Rb}$. Low lying excited states are produced by imprinting a local phase onto the BEC wave function. By monitoring the evolution of the density profile we study the successive dynamics of the wave function. The evolution of density minima traveling at a smaller velocity than the speed of sound in the trapped condensate is observed. By comparison to analytical and numerical solutions of the 3D Gross-Pitaevskii equation for our experimental conditions we identify these density minima to be moving dark solitons.
In our experiment, a highly anisotropic confining potential leading to a strongly elongated shape of the condensate allows us to be close to the (quasi) 1D situation where dark solitons are expected to be dynamically stable [9]. Parallel to this work, solitonlike states in nearly spherical BEC's of ${ }^{23} \mathrm{Na}$ are investigated at NIST [14].

Dark solitons in matter waves are characterized by a local density minimum and a sharp phase gradient of the wave function at the position of the minimum (see Figs. 1a and $1 \mathrm{~b}$ ). The shape of the soliton does not change. This is due to the balance between the repulsive interparticle interaction trying to reduce the minimum and the phase gradient trying to enhance it. The macroscopic wave function of a dark soliton in a cylindrical harmonic trap forms a plane of minimum density (DS plane) perpendicular to the symmetry axis of the confining potential. Thus, the corresponding density distribution shows a minimum at the DS plane with a width of the order of the (local) correlation length. A dark soliton in an homogeneous BEC of density $n_{0}$ is described by the wave function (see [10], and references therein)

$$
\begin{aligned}
\Psi_{k}(x)=\sqrt{n_{0}}( & i \frac{v_{k}}{c_{s}}+\sqrt{1-\frac{v_{k}^{2}}{c_{s}^{2}}} \\
& \left.\times \tanh \left[\frac{x-x_{k}}{l_{0}} \sqrt{1-\frac{v_{k}^{2}}{c_{s}^{2}}}\right]\right),
\end{aligned}
$$

with the position $x_{k}$ and velocity $v_{k}$ of the DS plane, the correlation length $l_{0}=\left(4 \pi a n_{0}\right)^{-1 / 2}$, and the speed of sound $c_{s}=\sqrt{4 \pi a n_{0}} \hbar / m$, where $m$ is the atom mass.

For $T=0$ in $1 \mathrm{D}$, dark solitons are stable. In this case, only solitons with zero velocity in the trap center do not move, otherwise they oscillate along the trap axis [8]. However, in 3D at finite $T$, dark solitons exhibit thermodynamic and dynamical instabilities. The interaction of the soliton with the thermal cloud causes dissipation which accelerates the soliton. Ultimately, it reaches the speed of sound and disappears [10]. The dynamical instability originates from the transfer of the 
(a)

$\left|\Psi_{\mathrm{k}}\right|^{2}$

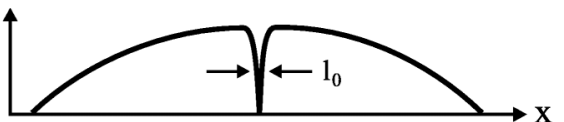

(b)

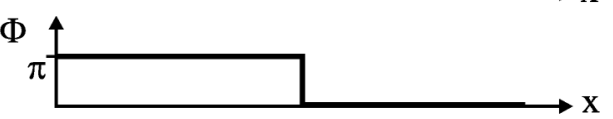

(c)

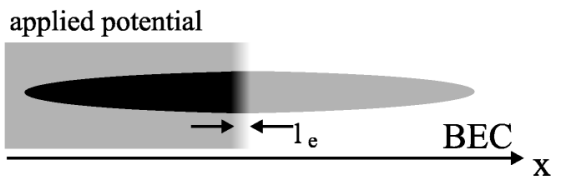

FIG. 1. Density distribution (a) and phase distribution (b) of a dark soliton state with $\Delta \phi=\pi$. The density minimum has a width $\sim l_{0}$. The scheme for the generation of dark solitons by phase imprinting is shown in (c), where $l_{e}$ is the width of the potential edge.

(axial) soliton energy to the radial degrees of freedom and leads to the undulation of the DS plane, and ultimately to the destruction of the soliton. This instability is essentially suppressed for solitons in cigar-shaped traps with a strong radial confinement [9], such as in our experiment [15].

As can be seen from Eq. (1), the local phase of the dark soliton wave function varies only in the vicinity of the DS plane, $x \approx x_{k}$, and is constant in the outer regions, with a phase difference $\Delta \phi$ between the parts left and right to the DS plane (see, e.g., Fig. 1b).

To generate dark solitons we apply the method of phase imprinting [13], which allows one also to create vortices and other textures in BEC's. We apply a homogeneous potential $U_{\text {int }}$, generated by the dipole potential of a far detuned laser beam, to one-half of the condensate wave function (Fig. 1c). The potential is pulsed on for a time $t_{p}$, such that the wave function locally acquires an additional phase factor $e^{-i \Delta \phi}$, with $\Delta \phi=U_{\text {int }} t_{p} / \hbar \sim$ $\pi$. The pulse duration is chosen to be short compared to the correlation time of the condensate, $t_{c}=\hbar / \mu$, where $\mu$ is the chemical potential. This ensures that the effect of the light pulse is mainly a change of the phase of the $\mathrm{BEC}$, whereas changes of the density during this time can be neglected. Note, however, that due to the imprinted phase, at larger times one expects an adjustment of the phase and density distribution in the condensate. This will lead to the formation of a dark soliton and also to additional structures as discussed below.

In our experimental setup (see [16]), condensates containing typically $1.5 \times 10^{5}$ atoms in the $\left(F=2, m_{F}=\right.$ $+2)$ state, with less than $10 \%$ of the atoms being in the thermal cloud, are produced every $20 \mathrm{~s}$. The fundamental frequencies of our static magnetic trap are $\omega_{x}=2 \pi \times$ $14 \mathrm{~Hz}$ and $\omega_{\perp}=2 \pi \times 425 \mathrm{~Hz}$ along the axial and radial directions, respectively. The condensates are cigar-shaped with the long axis ( $x$ axis) oriented horizontally.

For the phase imprinting potential $U_{\text {int }}$, a blue detuned, far off resonant laser field $(\lambda=532 \mathrm{~nm})$ of intensity $I \approx 20 \mathrm{~W} / \mathrm{mm}^{2}$ pulsed for a time $t_{p}=20 \mu$ s results in a phase shift $\Delta \phi$ of the order of $\pi$ [17]. Spontaneous processes can be totally neglected. A high quality optical system is used to image an intensity profile to the $\mathrm{BEC}$, nearly corresponding to a step function with a width of the edge, $l_{e}$, smaller than $3 \mu \mathrm{m}$ (see Fig. 1c). The corresponding potential gradient leads to a force transferring momentum locally to the wave function and supporting the creation of a density minimum at the position of the DS plane for the dark soliton. Note that also the velocity of the soliton depends on $l_{e}$ (see Fig. 3c).

After applying the dipole potential we let the atoms evolve within the magnetic trap for a variable time $t_{\mathrm{ev}}$. We then release the BEC from the trap (switched off within $200 \mu \mathrm{s}$ ) and take an absorption image of the density distribution after a time of flight $t_{\mathrm{TOF}}=4 \mathrm{~ms}$ (reducing the density in order to get a good signal-to-noise ratio in the images).

In a series of measurements we have studied the creation and successive dynamics of dark solitons as a function of the evolution time and the imprinted phase. Figure 2 shows density profiles of the atomic clouds for different evolution times in the magnetic trap, $t_{\mathrm{ev}}$. The potential $U_{\text {int }}$ has been applied to the part of the BEC with $x<0$. For this measurement the potential strength was estimated to correspond to a phase shift of $\sim \pi$.

For short evolution times the density profile of the BEC shows a pronounced minimum (contrast about $40 \%$ ). After a time of typically $t_{\mathrm{ev}} \approx 1.5 \mathrm{~ms}$ a second minimum appears. Both minima (contrast about 20\% each) travel in opposite directions and in general with different velocities. Figure 3a shows the evolution of these two minima in comparison to theoretical results obtained numerically from the 3D Gross-Pitaevskii equation.

One of the most important results of this work is that both structures move with velocities which are smaller than the speed of sound $\left(c_{s} \approx 3.7 \mathrm{~mm} / \mathrm{s}\right.$ for our parameters) and depend on the applied phase shift. Therefore, the observed structures are different from sound waves in a condensate as first observed at MIT [18]. We identify the minimum moving slowly in the negative $x$ direction to be the DS plane of a dark soliton.

We have performed a series of measurements with different parameter sets for $l_{e}$ and the product of laser

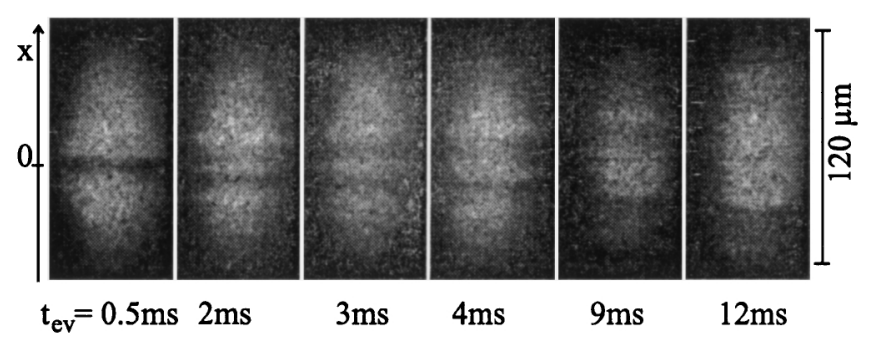

FIG. 2. Absorption images of BEC's with kink-wise structures propagating in the direction of the long condensate axis, for different evolution times in the magnetic trap, $t_{\mathrm{ev}}$. $\left(\Delta \phi \sim \pi, N \approx 1.5 \times 10^{5}\right.$, and $\left.t_{\mathrm{TOF}}=4 \mathrm{~ms}\right)$. 

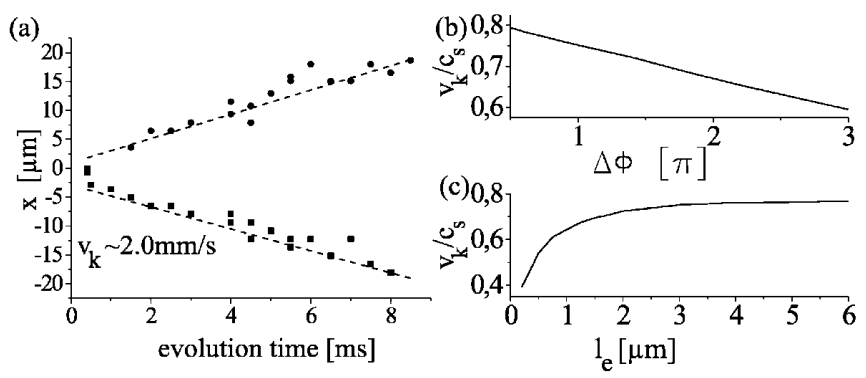

FIG. 3. (a) Position of the experimentally observed density minima versus evolution time in the magnetic trap for $\Delta \phi \sim$ $0.5 \pi$. The dashed lines show results from the 3D simulation for $N=5 \times 10^{4}, l_{e}=3 \mu \mathrm{m}$, and $\Delta \phi=2 \pi / 3$. (b) and (c) show the dependence of $v_{k} / c_{s}$ on the imprinted phase (for $l_{e}=3 \mu \mathrm{m}$ ) and on $l_{e}$ (for $\Delta \phi=\pi$ ) obtained numerically in quasi-1D simulations (see [16]) (with $N=1.5 \times 10^{5}$ ).

intensity and imprinting time. The velocity of the dark soliton could thereby be varied between $v_{k}=2.0 \mathrm{~mm} / \mathrm{s}$ (Fig. 3a) and $v_{k}=3.0 \mathrm{~mm} / \mathrm{s}$. For fixed $l_{e}$, the velocity $v_{k}$ decreases with increasing $\Delta \phi$. An increase of $\Delta \phi \sim$ $\pi / 2$ by a factor of 1.5 results in decreasing $v_{k}$ by $10 \%$, in agreement with theoretical results (see Figs. $3 b$ and $3 c$ ). For the significantly reduced imprinted phase we did not observe any dark soliton structures. For higher imprinted phase values more complex structures with several density minima were observed.

In addition to the dark soliton, the dipole potential creates a density wave traveling in the positive $x$ direction with a velocity close to $c_{s}$. After opening the trap, a complex dynamics results in the appearance of a second minimum behind the density wave as explained below.

To understand the generation of dark solitons and their behavior in the initial stages of the evolution we have performed numerical simulations of the 3D Gross-Pitaevskii equation. Computing time limitations have restricted our studies to atom numbers below $5 \times 10^{4}$. The simulations describe well the case $T=0$, but ignore the effects of thermodynamic instability. The latter was analyzed by using a generalization of the theory of Ref. [10].

Our theoretical findings are summarized as follows:

(1) The results of the simulations agree well with the experimental observations. After applying a phase changing potential, a dark soliton moves in the negative $x$ direction (Fig. 4a). The generation of the soliton by the phase imprinting method is accompanied by a density wave moving in the opposite direction. The maximum of the density wave travels with a velocity $\sim c_{s}$, independently of the values of $\Delta \phi$ and $l_{e}$. A characteristic time for the creation of the soliton is of the order of $l_{0} / c_{s}$ and in our case it does not exceed fractions of ms. Note that after this time the soliton-related phase slip in the wave function is affected by a complex dynamics of the soliton generation and will be different from the imprinted $\Delta \phi$.

(2) For a fixed $l_{e}$, the increase of $\Delta \phi$ from $\pi$ to $2 \pi, 3 \pi, \ldots$, leads to the creation of double, triple, etc.,
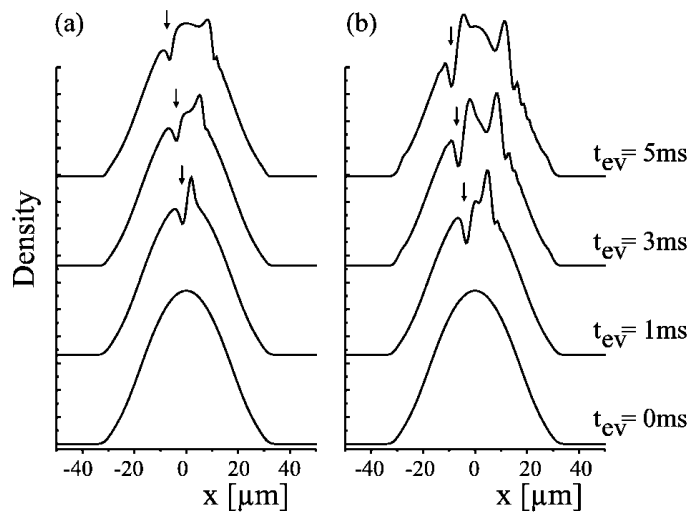

FIG. 4. Evolution of the density distribution obtained numerically from the 3D simulations for $N=10^{4}$ and $\Delta \phi=2 \pi / 3$. (a) Evolution inside the magnetic trap for different $t_{\mathrm{ev}}$; the dark soliton is marked by an arrow. (b) After $t_{\mathrm{TOF}}=4 \mathrm{~ms}$, a second density minimum is observed behind the density wave.

solitons. BEC's with several dark solitons were also observed experimentally and are currently investigated in detail.

(3) The initial soliton velocity decreases as $l_{e} \rightarrow 0$ (Fig. 3c). As observed experimentally, typical soliton velocities (for $l_{e}<3 \mu \mathrm{m}$ ) are smaller than $c_{s}$ and grow with the number of atoms. Velocities of the accompanying density waves are close to $c_{s}$. These waves move away from the center of the trap, broaden, and eventually vanish (Fig. 4). This is in contrast to solitons which are expected to oscillate in the trap, retaining their width and absolute depth. However, the observation of these oscillations would require longer lifetimes of the solitons (limited by dissipation to $\sim 10 \mathrm{~ms}$; see below). Within this time scale we find no signatures of dynamical instability and reveal only a moderate change $(<10 \%)$ of the soliton velocity, in agreement with our experiments.

(4) The situation changes after opening the trap and allowing the condensate to ballistically expand in the radial direction. The simulation shows that the soliton velocity drops down rapidly, while its width grows. To understand this aspect we have used a scaling approach, similar to that of $[19,20]$, for the radial ballistic expansion of an infinitely elongated condensate containing a moving kink. This approach (valid for $\left.\omega_{\perp}^{-1} \leq t_{\mathrm{TOF}} \leq \mu / \hbar \omega_{\perp}^{2}\right)$ predicts a soliton velocity $v_{k}\left(t_{\mathrm{TOF}}\right) \approx v_{k}\left(t_{\mathrm{ev}}\right) \ln \left(2 \omega_{\perp} t_{\mathrm{TOF}}\right) / \omega_{\perp} t_{\mathrm{TOF}}$, where $v_{k}\left(t_{\mathrm{ev}}\right)$ is the soliton velocity at $t_{\mathrm{ev}}$ just before switching off the trap. This result agrees very well with both experimental data and numerical simulations for a finite size BEC. Moreover, in a short time $t_{\mathrm{TOF}} \simeq \omega_{\perp}^{-1}$ after switching off the trap, the density develops a second minimum located between the density wave and the dark soliton. This minimum has a width and depth comparable to those of the dark soliton (see Fig. 4b). Its position regarded as a function of $t_{\mathrm{ev}}$ moves with a constant velocity similar to that of the soliton. The creation of this second minimum is a coherent phenomenon and can be attributed to a 
dynamically acquired phase of the wave function in the region between the density wave and the dark soliton.

The results of the experiment also show clear signatures of the presence of dissipation originating from the interaction of the soliton with the thermal cloud. We observe a decrease of the contrast of the soliton by $\approx 50 \%$ on a time scale of $10 \mathrm{~ms}$. This is in contradiction with the nondissipative dynamics, where the contrast should even increase for the soliton moving away from the trap center. The soliton energy is then proportional to $n_{0}^{3 / 2}\left(x_{k}\right)\left[1-v_{k}^{2} / c_{s}^{2}\left(x_{k}\right)\right]^{3 / 2}$ (see [10]) and should remain constant. This leads to a constant absolute depth of the soliton and hence gives the contrast proportional to $\left[1-v_{k}^{2} / c_{s}^{2}\left(x_{k}\right)\right] \sim n_{0}\left(x_{k}\right)^{-1}$. The decrease of the soliton contrast can therefore be explained only by the presence of dissipation decreasing the soliton energy. As the lifetime of the soliton is sensitive to the gas temperature, the studies of dissipative dynamics of solitons will offer a unique possibility for thermometry of BEC's in the conditions where the thermal cloud is not discernible.

In conclusion, we have created dark solitons by a phase imprinting method and studied their dynamics. A detailed comparison to theory and numerical simulations allows us to identify the creation of dark solitons traveling with approximately constant velocity smaller than the speed of sound. The initial stages of the evolution and the radial ballistic expansion of the sample are well described by the $T=0$ approach which also shows the absence of dynamical instabilities. The decrease of the soliton contrast gives a clear signature of dissipation in the soliton dynamics.

For the study of dark solitons with even lower velocities, an initial density preparation of the BEC in the magnetic trap may be useful. This can be done, e.g., by applying adiabatically an additional blue detuned laser beam prior to the phase imprinting pulse. A promising attempt will be the realization of dark solitons in elongated dipole traps, e.g., generated by a blue detuned hollow laser beam [21]. With spin as an additional degree of freedom, the dynamics of dark solitons in condensates containing spin domains or spin waves can be studied.

We expect that the study of soliton structures in BEC's opens a new direction in atomic physics, related to nonlinear phenomena in a dissipative environment.

We thank D. Petrov and K. Rza̧żewski for fruitful discussions and K.-A. Suominen for help in the numerical work. This work is supported by SFB407 of the Deutsche Forschungsgemeinschaft. G.S. acknowledges support from the Humboldt-Foundation, the Dutch Foundation FOM, and the Russian Foundation for Basic Studies.

[1] M.H. Anderson, J.R. Ensher, M.R. Matthews, C.E.
Wieman, and E. A. Cornell, Science 269, 198 (1995); K. B. Davis, M.-O. Mewes, M. R. Andrews, N.J. van Druten, D. S. Durfee, D. M. Kurn, and W. Ketterle, Phys. Rev. Lett. 75, 3969 (1995); C. C. Bradley, C. A. Sackett, J. J. Tollett, and R. G. Hulet, Phys. Rev. Lett. 75, 1687 (1995); 78, 985 (1997); D. G. Fried, T.C. Killian, L. Willmann, D. Landhuis, S. C. Moss, D. Kleppner, and T. J. Greytak, Phys. Rev. Lett. 81, 3811 (1998).

[2] L. Deng, E. W. Hagley, J.W. En, M. Trippenbach, Y. Band, P. S. Julienne, J.E. Simsarian, K. Helmerson, S. L. Rolston, and W.D. Phillips, Nature (London) 398, 218 (1999).

[3] R. J. Donnely, Quantized Vortices in Helium II (Cambridge University Press, Cambridge, 1991).

[4] M. R. Matthews, B. P. Anderson, P.C. Haljan, D. S. Hall, C. E. Wieman, and E. A. Cornell, Phys. Rev. Lett. 83, 2498 (1999).

[5] Y.S. Kivshar and B. Luther-Davies, Phys. Rep. 298, 81 (1998).

[6] P. A. Ruprecht, M. J. Holland, K. Burnett, and M. Edwards, Phys. Rev. A 51, 4704 (1995).

[7] W. Zhang, D. F. Walls, and B. C. Sanders, Phys. Rev. Lett. 72, 60 (1994); W. P. Reinhardt and C. W. Clark, J. Phys. B 30, L785 (1997); A. D. Jackson, G. M. Kavoulakis, and C. J. Pethick, Phys. Rev. A 58, 2417 (1998).

[8] Th. Busch and J. Anglin, cond-mat/9809408.

[9] A.E. Muryshev, H.B. van Linden van den Heuvell, and G. V. Shlyapnikov, Phys. Rev. A 60, R2665 (1999).

[10] P. O. Fedichev, A.E. Muryshev, and G. V. Shlyapnikov, Phys. Rev. A 60, 3220 (1999).

[11] R. Dum, J. I. Cirac, M. Lewenstein, and P. Zoller, Phys. Rev. Lett. 80, 2972 (1998).

[12] T.F. Scott, R. J. Ballagh, and K. Burnett, J. Phys. B 31, L329 (1998).

[13] Ł. Dobrek, M. Gajda, M. Lewenstein, K. Sengstock, G. Birkl, and W. Ertmer, Phys. Rev. A 60, R3381 (1999).

[14] W.D. Phillips and K. Helmerson (private communication).

[15] In our case the solitons move with a velocity $\sim 0.5 c_{s}$ and are dynamically stable already at a smaller radial confinement than in the case of standing solitons.

[16] K. Bongs, S. Burger, G. Birkl, K. Sengstock, W. Ertmer, K. Rzążewski, A. Sanpera, and M. Lewenstein, Phys. Rev. Lett. 83, 3577 (1999).

[17] The imprinted phase is estimated from measured laser parameters and tables of atomic data. In addition, the onset (contrast $>10 \%$ ) for dark soliton creation was measured to correspond to $\Delta \phi \sim 0.5 \pi$, in agreement with the theoretical results.

[18] M. R. Andrews, D. M. Kurn, H.-J. Miesner, D. S. Durfee, C. G. Townsend, S. Inouye, and W. Ketterle, Phys. Rev. Lett. 79, 553 (1997).

[19] Y. Kagan, E.L. Surkov, and G. V. Shlyapnikov, Phys. Rev. A 54, R1753 (1996); 55, R18 (1997).

[20] Y. Castin and R. Dum, Phys. Rev. Lett. 77, 5315 (1996).

[21] S. Burger, K. Bongs, K. Sengstock, and W. Ertmer, Proceedings of the Internation School of Quantum Electronics, 27th Course, Erice, 1999, edited by A. Aspect and M. Inguscio (Kluwer Academic, London, to be published). 